\title{
Production des risques dits «naturels » dans les grands centres urbains du Cameroun
}

\author{
Frédéric Saha ${ }^{1, *}$, Dagobert Tchio Nkemta ${ }^{2}$, Mesmin Tchindjang ${ }^{3}$, Éric Voundi ${ }^{4}$ \\ et Philippes Mbevo Fendoung 5
}

${ }^{1}$ Géographie, Université de Yaoundé 1, Unité de recherche et de formation doctorale en science humaine et sociale, Yaoundé, Cameroun

${ }^{2}$ Urbanisme, Université Toulouse-Jean-Jaurès, INP-ENSAT, Auzeville-Tolosane, France

${ }^{3}$ Géographie/environnement, Université de Yaoundé 1, Yaoundé, Cameroun

${ }^{4}$ Géographie, Global Mapping and Environmental Monitoring (GMEM), Yaoundé, Cameroun

${ }^{5}$ Géomatique, Global Mapping and Environmental Monitoring (GMEM), Yaoundé, Cameroun

Reçu le 14 décembre 2015. Accepté le 14 juin 2018

Dans un monde de plus en plus urbanisé, la question des risques naturels en ville est porteuse de forts enjeux. Mais de quoi parle-t-on? Quelle est cette « Nature » à l'origine de ce risque ? Ce risque n'est-il pas avant tout le produit de sociétés, et ce à de multiples échelles ? Analyser les risques naturels en ville implique de se référer à un espace, à une époque, à un contexte. L'approche illustrative représente alors un moyen d'aborder les modalités de la production du risque afin d'identifier de potentiels leviers d'action et de prévention. À partir de quatre villes illustrant la diversité du Cameroun, prenant en compte le contexte géographique, historique et politique, les auteurs de cet article ont cherché à cerner les déterminants de la production du risque et leurs modalités d'expression. Pour autant, si des leviers existent pour agir, ils semblent encore difficilement accessibles.

La rédaction

Résumé - Cet article examine les facteurs de production des risques dits «naturels» dans les grandes villes du Cameroun. D'une approche historique, il ressort que l'établissement d'agglomérations dans des sites d'aménagement difficile est à la base du problème. Le manque de ressources ainsi que la forte croissance démographique qui caractérisent les pays les moins avancés (PMA) en général et le Cameroun en particulier sont du point de vue anthropique les causes de la forte sensibilité aux risques naturels. Il se pose aussi le problème de l'ambiguité du système foncier qui n'aide pas à la maîtrise de l'espace. L'incapacité des autorités et de la protection civile à gérer l'espace urbain consolide la vulnérabilité. Les changements climatiques diminuent la perception et l'acceptation des risques par les populations et augmentent leur fréquence.

Mots-clés : Cameroun / protection civile / risques naturels / villes / vulnérabilité

\begin{abstract}
Production of so-called "natural" risks in major urban centers of Cameroon. This paper looks into the causes of the production of so-called "natural" risks in major cities of Cameroon. From a historical approach, it appears that the establishment of settlements in uneven sites (steep slopes, swampy areas, rugged plateaus, coastal plains) is at the root of the problem. The lack of resources that characterizes the Least Developed Countries in general and Cameroon in particular, coupled with a high population growth are some anthropogenic factors expressing the high sensitivity to natural hazards. It also raises the problem of the ambiguity of the land tenure system with the intervention of non-institutional actors (population and traditional rulers) which does not help in a production of adequate plots for construction. The inability of urban authorities and the administrative services to take good care of the urbanization
\end{abstract}

\footnotetext{
*Auteur correspondant : fredericsaha@yahoo.fr
} 
process multiply the vulnerability. Climate changes (increase of temperature and rainfall disturbances) reinforce hazards, increase the frequency, and reduce risk perception and acceptance of the population. Floods, landslides, rock falls, drought, coastal erosion and gullying are main natural hazards that cause important damages in Cameroonian cities every year. It is important to find adequate solutions including all key actors and parameters to minimize as far as possible losses.

Keywords: Cameroon / civil protection / natural hazards / cities / vulnerability

Le risque naturel est le produit d'un phénomène naturel dit aléa et de la vulnérabilité des personnes et des biens exposés (Wisner et al., 2003) ${ }^{1}$. La vulnérabilité dépend de la sensibilité et de l'exposition à l'aléa, et traduit la propension à subir les dommages. Au vu du rôle déterminant de la vulnérabilité qui résulte des écueils de la construction du territoire ou mieux des défaillances dans la mise en commun des différents ressorts territoriaux, les risques naturels n'ont de naturel que leur nom (Martin, 2006). Il s'agit donc de risques « dits naturels », désignés «risques naturels » dans la suite de cette contribution pour des raisons de commodité. En fonction de l'origine naturelle du processus générateur de risque (aléa), on peut distinguer les risques géophysiques (tremblements de terre et éruptions volcaniques), les risques météorologiques et climatiques (cyclones tropicaux, tempêtes, températures extrêmes, sécheresses et feux de brousses), les risques hydrologiques (inondations) et les risques morphodynamiques comme les glissements de terrain. Ces risques font partie du fonctionnement normal de différents cycles qui caractérisent les éléments de la biosphère (Burton et al., 1978). Leurs caractères catastrophiques découlent de la multiplication des enjeux, les zones du monde présentant les plus fortes densités de populations et une importante mise en valeur économique étant les plus susceptibles. Durant la décennie 2003-2012, 215,49 millions de personnes en moyenne ont été victimes chaque année des catastrophes naturelles (Guha-Sapir et al., 2014). L'Asie, l'Amérique et l'Afrique sont par ordre décroissant les continents qui ont enregistré le plus grand nombre de catastrophes par an. Avec un ratio de 417916 victimes/catastrophe, l'Afrique arrive au second rang derrière l'Asie (1132967 victimes/catastrophe). Les autres continents présentent un taux moyen de 40467 victimes/catastrophe ${ }^{2}$. Cette forte disparité dans la sensibilité aux risques naturels interroge la préparation

\footnotetext{
${ }^{1}$ Cet article résulte de la synthèse de recherches d'étudiants en urbanisme et gestion de risques dans le cadre de la préparation de leurs mémoires de Master 2 à l'Université de Yaoundé 1 au Cameroun.

${ }^{2}$ En divisant le nombre moyen de victimes/an (215,49 millions) par le nombre moyen annuel de catastrophes (388), on obtient un taux moyen mondial de 555386 victimes/catastrophe (Guha-Sapir et al., 2014).
}

de chaque communauté humaine aux risques de catastrophes (Baird et al., 1975; Veyret et Meschinet de Richemond, 2003). L'Europe et l'Amérique du Nord, grâce à certains atouts inhérents à la maîtrise technologique et à une certaine rigueur dans la planification de l'aménagement du territoire, minimisent un tant soit peu leurs vulnérabilités. Les pays asiatiques et africains les plus touchés par la pauvreté sont susceptibles de voir des menaces apparemment mineures se transformer en catastrophes.

Le cas du continent africain est d'autant plus préoccupant qu'il présente ces dernières décennies le taux de croissance démographique le plus fort au monde (Banque mondiale). Cette croissance est majoritairement absorbée par les centres urbains qui s'illustrent par une criante incapacité d'accueil. Ledoux (1995) souligne que dans les pays en développement, les populations s'installent en masse en milieu urbain au détriment des capacités d'accueil déjà très limitées des infrastructures. D'une part, les nouveaux arrivants, souvent pauvres, contribuent à la densification de l'habitat dans les «vieux quartiers» en occupant les bas-fonds inondables et les pentes abruptes peu convoitées. D'autre part, ils s'installent dans les marges urbaines dénuées de la présence minimale de services de base et ne prenant pas en compte les caractéristiques des milieux, générant ainsi un phénomène de pseudo-urbanisation. La conséquence en est in fine le développement de vastes bidonvilles où les populations sont extrêmement vulnérables aux risques de catastrophe. Au Cameroun, l'armature urbaine laisse voir un foisonnement de villes de fait, résultat d'importantes lacunes dans la planification. Nombre de ces grandes agglomérations enregistrent chaque année des aléas coûteux en vies humaines et en biens matériels.

Quels sont les facteurs qui expliquent la production des risques naturels dans les grandes agglomérations $\mathrm{du}$ Cameroun? Cet article retrace brièvement les grands moments du processus d'urbanisation au Cameroun en mettant en exergue les limites d'accueil des sites des grandes villes. Puis un accent particulier est mis sur les facteurs anthropiques qui se combinent aux limites de situation pour transformer les villes camerounaises en structures très susceptibles aux catastrophes naturelles. 


\section{Mise en contexte et objectif de l'étude}

Les villes sont difficilement définissables; elles présentent toutefois quelques caractères communs. Par exemple, Salem (1995) dit que la ville est un espace dense, hétérogène, artificialisé, doté de nombreuses infrastructures et générant d'importantes densités de populations exerçant dans une diversité d'activités. L'accroissement naturel et les migrations sont les moteurs de la croissance démographique des villes en Afrique (Antoine, 1990). Au niveau spatial, peu de critères ont présidé au choix des sites des grandes villes dans le monde. L'histoire de l'humanité ayant été rythmée par des guerres, les hommes se sont installés dans des sites de défense (zones montagneuses, boucles de méandres, bordures de falaises, buttes isolées...) pour se retrancher des ennemis. D'autres communautés ont porté leurs choix sur des vallées donnant accès à l'eau et aux terres fertiles et drainées. Certaines villes sont établies sur la côte pour profiter de l'ouverture au monde, d'autres dans des zones où abondent des ressources naturelles. Confrontés à la croissance démographique, de nombreux sites ont vu leurs capacités d'accueil réduites (Santos, 1971). Le manque de logements et d'emplois, les problèmes d'assainissement et la bidonvilisation comptent parmi les difficultés qui se posent avec plus d'acuité dans les villes des pays en développement (Hossenlopp, 1971; Savane et Antoine, 1989; Olvera et al., 2002). Ces difficultés intensifient la vulnérabilité des populations et de leurs biens face aux risques de catastrophes. Tchindjang (2012) et November (1994) parlent de coévolution ou de l'inhérence du risque naturel à l'évolution urbaine.

$\mathrm{Au}$ Cameroun, une vision réductrice ne prenant en compte que la dimension administrative au détriment des autres caractéristiques citées plus haut définit la ville comme tout chef-lieu de circonscription administrative (BUCREP, 2010), masquant ainsi une extrême diversité de taille, de forme, de statut et donc d'enjeux, notamment par rapport à la prise en compte des risques naturels ou non. Sur le plan historique, on peut distinguer les vieilles villes souvent d'origine coloniale et les créations récentes. Les Allemands avaient aménagé des postes militaires (Mora, Adamaoua, Banyo, Bamoun, Édéa, Yaoundé, Kribi, Ebolowa, Lolodorf et Douala) qui correspondaient aux dix circonscriptions administratives que comptait le Cameroun colonial (Mveng, 1984). Ces postes militaires furent progressivement dotés de services sociaux et d'infrastructures de production. Lorsque qu'en 1916 la France et la Grande-Bretagne se partagèrent le Cameroun, le développement polynucléaire fut promu dans la partie occidentale par la Grande-Bretagne (Fombe et Balgah, 2010). Dans la partie orientale, les Français se sont appuyés sur les centres existants pour développer d'autres pôles en fonction des préoccupations économiques et administratives. Au lendemain des indépendances, plusieurs autres agglomérations ont progressivement gagné de l'importance grâce à leurs croissances spatiales et démographiques, les découpages et redécoupages administratifs jouant le rôle de catalyseurs d'urbanisation. Même si les pouvoirs publics ont consacré des moyens considérables à l'aménagement urbain, le rythme très élevé de la croissance spatiale et démographique couplé à la crise économique a dilué ces efforts. On remarque une discrimination spatiale; d'un côté les quartiers huppés qui bénéficient d'une attention constante, de l'autre côté les quartiers délaissés (Assako Assako, 1995; Cameroon Engineering, 2008; Olinga Olinga, 2012; Fombe et Balgah, 2010; Saha, 2013). Dans les villes de plateaux, les mouvements de terrain mettent en danger la sécurité des hommes et des biens installés en zones de pente, tandis que dans les quartiers en vallée comme dans les agglomérations de plaine, ce sont les inondations. C'est fort de ces constats que ce travail interroge les causes de l'intensification de la menace des risques naturels sur les grandes agglomérations du Cameroun.

\section{Cadre de l'étude et démarche méthodologique}

\section{Cadre de l'étude}

Le Cameroun est un pays d'A frique centrale. Il est situé au nord de l'équateur, entre les $8^{\mathrm{e}}$ et $16^{\mathrm{e}}$ degrés de longitude est et les $2^{\mathrm{e}}$ et $13^{\mathrm{e}}$ degrés de latitude nord (Fig. 1). Le pays comptait 21917602 habitants au $1^{\mathrm{er}}$ janvier 2015 dont environ $50 \%$ d'urbains, selon l'estimation du troisième recensement général de la population et de l'habitat (BUCREP, 2010). Il partage une longue frontière $(1690 \mathrm{~km})$ avec le Nigeria, pays le plus peuplé d'Afrique. Ces dernières années, le Cameroun subit un important flux de migrants issus notamment du Nigeria, du Tchad et de la République centrafricaine (RCA), tous confrontés à des problèmes de sécurité.

Sur le plan physique, on distingue globalement deux principaux domaines climatiques : le domaine équatorial et le domaine tropical (Suchel, 1988). Le domaine tropical s'étend au-delà du $6^{\mathrm{e}}$ parallèle. On y distingue plusieurs nuances. Au Sud, sur le plateau de l'Adamaoua polarisé par la ville de Ngaoundéré, il est humide. La ville de Maroua (796 $\mathrm{mm}$ de précipitations/an) se situe aux confins nord de la nuance soudano-sahélienne humide. Dans la ville de Kousséri plus au Nord, on a tendance à passer du semi-aride à l'aride. L'ensemble du domaine tropical où seulement $28,83 \%^{3}$ de la population vit en ville, est moins urbanisé. Les grandes agglomérations de cette partie du Cameroun subissent

\footnotetext{
$\overline{{ }^{3} \text { Ce taux est de } 51,28 \%}$ dans la partie équatoriale, pour une moyenne nationale de 48,8\% (RGPH 3).
} 
principalement les extrêmes climatiques, notamment les sécheresses et les inondations. Le domaine équatorial, également très diversifié, couvre toute la partie au Sud. Le relief, la continentalité, la végétation contribuent à la mise en place de plusieurs nuances climatiques où les risques naturels s'expriment de différentes manières. Le risque d'inondation est la principale menace d'origine naturelle sur les villes équatoriales. La récurrence des glissements de terrain n'y est pas négligeable, notamment dans les villes des hautes terres. Sur le plan hydrologique le Cameroun dispose $\mathrm{du}$ deuxième potentiel en Afrique. On y distingue cinq bassins hydrographiques : les bassins du lac Tchad, du Niger, de la Sanaga, du Congo et du Nyong et des fleuves côtiers. L'écoulement des eaux est calqué sur les saisons climatiques. Plusieurs grandes agglomérations urbaines sont traversées par les plus grands cours d'eau de ces différents bassins hydrographiques. C'est le cas de Douala avec le Wouri et de Garoua avec la Bénoué. En période de crues, certains quartiers sont immergés du fait de leur proximité avec le lit mineur du cours d'eau. Le Cameroun est constitué à $60 \%$ de hautes terres (le plateau sud-camerounais, le plateau de l'Adamaoua et les hautes terres de l'Ouest). On y retrouve aussi des plaines, notamment la plaine côtière et celle du lac Tchad au Nord. Les villes sont disséminées dans toutes ces unités topographiques; aucune n'est à l'abri des risques naturels (Tchindjang, 2012).

Compté parmi les PMA, le Cameroun présente une incidence de la pauvreté d'environ $37,5 \%$ et un indice de développement humain (IDH) de 0,518, ce qui le classe $153^{\mathrm{e}}$ sur 188 (PNUD, 2016). Les activités économiques se structurent autour des secteurs primaire $(44,8 \%$ du PIB), secondaire (17\% du PIB) et tertiaire $(38,2 \%$ du PIB). L'agriculture occupe $45,3 \%$ de la population active et $88,6 \%$ de l'ensemble des activités économiques sont mal formalisées ou informelles, selon les chiffres de l'Institut national de la statistique en 2015.

\section{Démarche méthodologique}

Les données de cette étude proviennent d'une analyse des travaux de recherche (publications scientifiques et littérature grise) sur les risques naturels dans les grandes villes du Cameroun. L'augmentation des pertes (vies humaines, traumatismes, destruction des infrastructures, etc.) liées aux catastrophes dans certains quartiers explique le foisonnement des études universitaires et des recherches commanditées par le gouvernement et des institutions internationales intervenant dans le pays (Thouret et D'Ercole, 2009; Tchindjang, 2012; Anougue Tonfack et al., 2013; UNISDR, 2015). Les auteurs s'appuient aussi et surtout sur des enquêtes sociales et économiques qu'ils ont conduites dans le cadre de la rédaction de leurs mémoires de fin de second cycle (Saha, 2013 ; Tchio Nkemta, 2014; Mbevo Fendoung, 2016; Voundi, 2016).

De par sa situation en zone de hautes terres caractérisée par un relief très accidenté, la ville de Bamenda illustre la problématique des risques affectant les villes des plateaux au Cameroun. Grâce à 172 questionnaires distribués à certains ménages choisis de façon aléatoire dans les quartiers à risque, l'historique des risques naturels dans cette ville a pu être retracé. En outre, des questions et des observations sur le cadre de vie et de travail des populations ont permis de hiérarchiser les facteurs de vulnérabilité tant naturels qu'anthropiques. La même approche à Yaoundé, notamment dans le quartier Mvog-Ada, pour évaluer le risque d'inondation au lieu-dit Fanta Citron, a permis de faire des propositions en faveur de la rénovation et/ou de la restructuration urbaine.

Cette étude tire aussi profit des plans de développement urbain (PDU) de certaines villes (Douala, Yaoundé, Bamenda, Garoua) pour établir le diagnostic socioéconomique des risques naturels en fonction des prévisions fixées. L'évaluation de la contribution des aléas climatiques au renforcement des catastrophes s'appuie sur les données de températures et de précipitations des cinq dernières décennies dans les stations installées dans les villes choisies. Ces données ont été mises à notre disposition par la Direction de la météorologie nationale ou par les délégations régionales des transports.

La mise en valeur de l'espace urbain est observée sur des images Google Earth. La possession d'une image Quick Bird de très haute résolution sur la ville de Yaoundé facilite la visualisation de l'occupation de l'espace urbain. La modélisation topographique et hydrologique sur des images ASTER et/ou SRTM ${ }^{4}$ laisse voir des contingences naturelles qui pèsent sur l'extension spatiale et l'orientation des fronts d'urbanisation. Le logiciel libre SIG (système d'information géographique) QGIS a permis de tracer les contours puis de calculer les altitudes et les pentes pour circonscrire les zones les plus à risques.

Le cadre réglementaire nous est donné par la loi $n^{\circ} 2004 / 003$ du 21 avril 2004 régissant l'urbanisme au Cameroun. Ce texte oriente l'aménagement en milieu urbain. Il fixe les règles relatives aux lotissements et aux constructions. Allusion est faite aux zones exposées aux risques naturels comme étant non aedificandi. Des sanctions et des opérations de correction sont aussi prévues (articles 53 à 58). D'autres lois sur la protection civile, notamment la loi n 86/016 du 6 décembre 1986

\footnotetext{
${ }^{4}$ Les images radar et SRTM (Shuttle Radar Topography Mission) présentent en plus des cordonnées x (longitude) et y (latitude) l'altitude (z) qui permet de faire les cartes topographiques.
} 


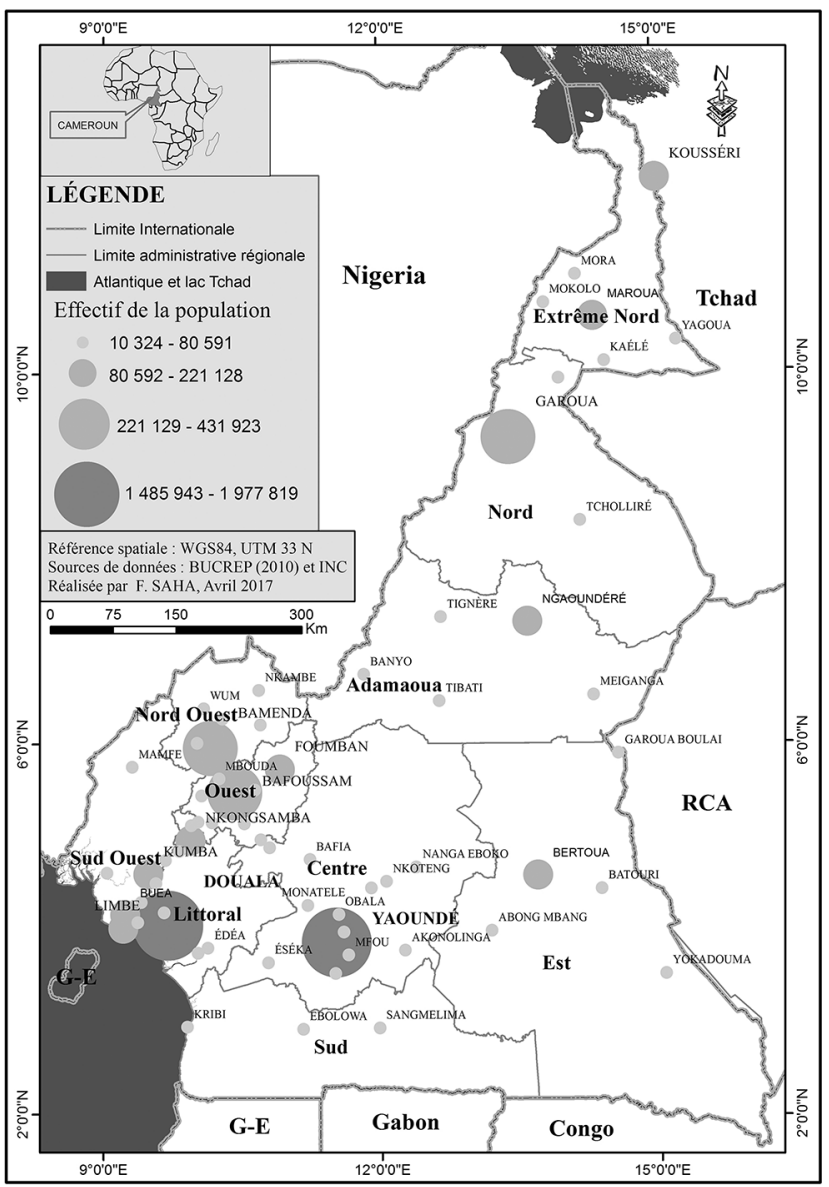

Fig. 1. Localisation du Cameroun en Afrique centrale et villes de plus de 10000 habitants.

(source: BUCREP, 2010; Institut national de cartographie [Cameroun]; réalisation: F. Saha).

portant réorganisation générale de la protection civile et le décret $n^{\circ} 98 / 031$ du 9 mars 1998 portant organisation des plans d'urgences et de secours en cas de catastrophe ou de risques majeurs, sont également exploitées.

\section{Présentation des résultats et analyse}

\section{Grandes agglomérations du Cameroun : des sites contraignants d'aménagement difficile}

Un regard exploratoire laisse voir que la plupart des grandes agglomérations du Cameroun sont localisées dans des sites contraignants, à urbanisation difficile. Les sites ayant connu un fort attrait démographique sont soit des plaines inondables soit des plateaux accidentés. La plaine côtière a connu une urbanisation fulgurante (Douala, Kribi, Limbé, etc.). Ces villes sont exposées aux inondations et à l'érosion côtière à l'image de bon nombre de villes côtières en Afrique: Abidjan (Bouabre
Gnoka, 2013), Libreville et Pointe-Noire (Mounganga, 2009), Nouakchott (Ould Sidi Cheikh et al., 2007), etc. De nombreuses villes intérieures et de la partie septentrionale sont localisées dans des plaines alluviales où les inondations font des dégâts en période de crues. Sur les plateaux, l'extension spatiale des villes est contrainte par la présence de vallées inondables et des pentes plus ou moins abruptes. Le cas de Bamenda dans les hautes terres de l'Ouest est assez illustratif. Bamenda est partagée en deux par un talus avec des pentes atteignant par endroits $30^{\circ}$ (Saha et Tchindjang, 2017). De part et d'autre, les vallées assurant le drainage des eaux sont soumises aux crues saisonnières. Yaoundé sur le plateau sud-camerounais s'étale sur un site similaire, voire plus contraignant. Assako Assako (1997) décrit le site de Yaoundé comme étant composé de sommets d'interfluves, de pentes et de fonds de vallées inondables. Le réseau hydrographique y est très dense (Franqueville, 1984). Les figures 2, 3, 4 et 5 présentent les sites de quelques grandes agglomérations du Cameroun en faisant ressortir leurs caractéristiques hydrogéomorphologiques.

\section{Facteurs de production des risques naturels dans les grandes agglomérations du Cameroun}

En plus des contraintes d'aménagement, force est de constater qu'au Cameroun, le processus d'urbanisation est mal maîtrisé, le régime foncier et les politiques publiques semblent incapables de faire appliquer la loi et/ ou tolèrent l'installation irrégulière des populations dans des espaces inappropriés. La forte croissance démographique et la pauvreté multiplient les vulnérabilités. Le caractère limité du cadre de gestion préventive et opérationnelle du risque pour l'ensemble du territoire augmente la susceptibilité des populations. Face au poids de facteurs anthropiques dans la construction du risque, on devrait parler de «risque d'origine naturelle» et non de risque naturel (Metzger et D’Ercole, 2011).

\section{Absence ou inefficacité de la planification urbaine}

L'aménagement, c'est-à-dire la façon de disposer et de répartir les activités, les équipements et les hommes en liaison avec l'espace disponible, est encore dans une phase de balbutiement au Cameroun. Le développement des grandes agglomérations est régi par le Code de l'urbanisme de 2004. Ce dernier fixe quelques principes permettant d'éviter les risques de catastrophe en milieu urbain : sont déclarés inconstructibles, sauf prescriptions spéciales, les terrains exposés à un risque naturel (inondation, érosion, éboulement, séisme, etc.); toute parcelle à bâtir doit permettre l'intervention des services 
de secours et de voirie (pompiers, assainissement, enlèvement des ordures ménagères, etc.); les documents de planification urbaine (plan directeur d'urbanisme, plan d'occupation des sols, plan de secteur, plan sommaire d'urbanisme) doivent permettre la prévention des risques naturels et des risques technologiques, ainsi que les pollutions et les nuisances de toute nature.

Dans les faits, un fossé existe entre la loi et les pratiques sur le terrain. Peu de communes urbaines et de communautés urbaines disposent de documents de planification. En outre, l'usage de plans de secteur est quasi inexistant. En conséquence, on constate des installations non réglementaires dans les bas-fonds et sur des pentes escarpées profitant du laxisme et des limites de la gestion domaniale. Dans le quartier Fanta Citron à Yaoundé, Tchio Nkemta (2014) remarque que $59 \%$ des populations accèdent à leurs domiciles en empruntant des passages tortueux et que $39 \%$ parviennent à leurs habitations par des pistes non carrossables, généralement noyées en saison de pluie. Seules $2 \%$ des habitations de la zone sont accessibles par voie carrossable.

\section{L'ambiguïté du régime foncier}

Le régime foncier du Cameroun est vieillissant. Issu des réformes du cadre établi par l'administration française avant les indépendances, il exclut le droit coutumier pourtant prédominant. Le statut légal subordonne la propriété foncière à la possession d'un titre foncier. La procédure d'obtention de cet acte est longue, coûteuse et trop codifiée, ce qui la rend complètement hors de portée d'une importante fraction de la population (Pettang, 1999). La grande diversité des acteurs impliqués dans la gestion foncière en milieu urbain est source de confusion et de complications. Entre les administrations (ministère de la Ville, ministère des Domaines, ministère de l'Administration territoriale), les collectivités territoriales décentralisées (les communautés urbaines, les mairies d'arrondissement de communes urbaines) et les institutions paraétatiques (la Société Immobilière du Cameroun, la Mission d'aménagement et d'équipement des terrains urbains et ruraux et le Crédit foncier du Cameroun), on constate des chevauchements de compétences et des attributions non assumées (Tchotsoua, 2007). Dans ces conditions, Njouonang Djomo (2012) relève que dans la ville de Yaoundé, plus de $51 \%$ de la population empreinte des voies illégales pour accéder à la terre. Il s'agit majoritairement des arrangements de gré à gré avec les propriétaires coutumiers. Pour cette raison, les doubles ventes, les problèmes de bornage ou de limites, la contestation du partage du patrimoine familial, les incompréhensions dans les opérations de gage, de location ou de vente sont très répandus. $\mathrm{La}$ situation d'insécurité foncière se généralisant, les populations rechignent à engager des investissements convenables dans l'aménagement et la construction de leurs habitations. Les opérations de restructuration urbaine dans les quartiers spontanés des villes de Douala et de Yaoundé représentent pour ces populations une réelle menace (Tchekote et Kaffo, 2012). Face à une poussée démographique sans cesse croissante, un début de solution serait l'allègement de la procédure d'obtention du titre foncier pour favoriser la formalisation de l'occupation de l'espace par les populations présentes et la prescription de mesures de construction en conformité avec les plans d'occupation du sol et la prévention des risques de catastrophes. Santos (1971) oppose l'urbanisation technico-économique des pays riches au développement urbain des pays du tiers-monde qu'elle qualifie de démographique.

\section{Le boom démographique urbain}

La population du Cameroun est passée de 7663246 habitants en 1976 à 10493655 habitants en 1987 puis à 17463836 habitants en 2005 pour être estimée à 21,9 millions ${ }^{5}$ au $1^{\mathrm{er}}$ janvier 2015 (BUCREP, 2010); soit un taux moyen de croissance démographique de 2,9\% par an. L'amélioration du cadre de vie par les efforts en matière d'hygiène, les progrès de santé maternelle et l'absence d'une politique démographique coercitive comptent parmi les raisons de cette croissance régulière de la population du Cameroun.

Les villes absorbent l'essentiel de cette croissance démographique. La part de la population urbaine est passée de $28,73 \%$ en 1976 à $38,04 \%$ en 1987 pour atteindre $48,80 \%$ en 2005. D'après les estimations de l'Institut national de la statistique du Cameroun (INS), ce taux dépasserait $50 \%$ en 2015. Douala et Yaoundé représentent près la moitié de l'ensemble de la population urbaine du Cameroun. Sur la base des données du troisième RGPH, la méthode de Huntsberger et la discrimination par progression arithmétique permettent de distinguer: un deuxième groupe de villes dont la population se situe entre 221129 et 431923 habitants (Bamenda, Bafoussam et Garoua). Il représente $11 \%$ de la population urbaine du Cameroun ; un troisième groupe (population comprise entre 80592 et 221128 habitants) constitué des villes de Maroua, Ngaoundéré, Kumba, Nkongsamba, Buéa, Kousséri, Bertoua, Limbé et Foumban. Il représente $17 \%$ de la population urbaine ; et un quatrième groupe composé de petites villes de 10324 à 80591 habitants, qui cumule $22 \%$ de l'ensemble de la population urbaine du pays (Fig. 1).

En moyenne, le taux de croissance démographique de ces villes (4,9\% entre 1976 et 2005) est supérieur au taux

\footnotetext{
${ }^{5}$ Le rapport du RGPH 3, pour une estimation/projection de la population du Cameroun en 2015, se fonde sur les données du recensement de 2005.
} 


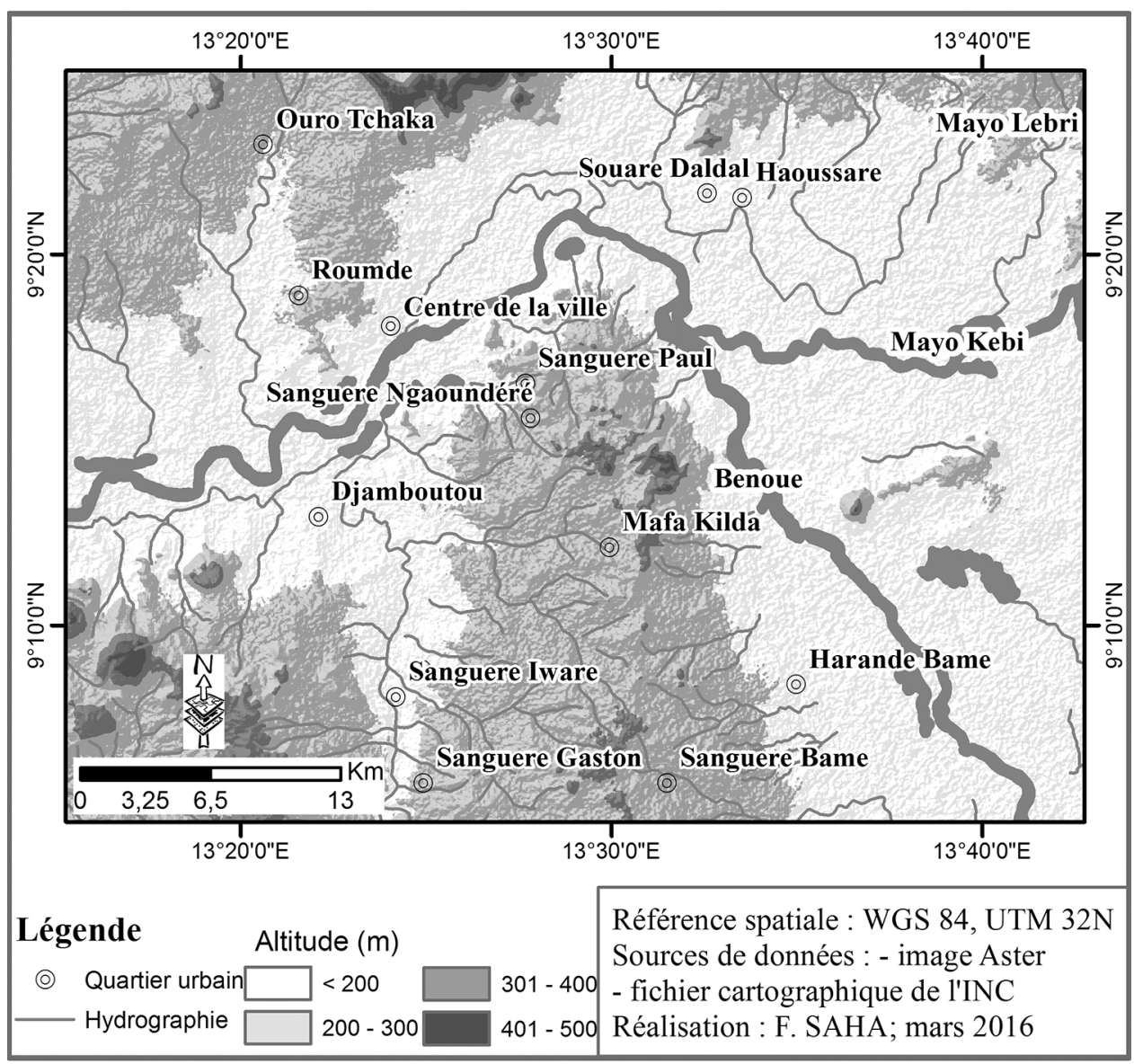

Fig. 2. Relief et contraintes du site de la ville de Garoua.

Garoua est la capitale de la région du nord du Cameroun. Cette ville présente des densités très variables. La moyenne est de 640 habitants $/ \mathrm{km}^{2}$. C'est à Garoua que le Mayo Kebi et la Bénoué confluent pour se diriger vers le Nigéria. Il s'agit d'une plaine alluviale avec des pentes quasiment nulles et des cours coulant en surface et dessinant de grands méandres. Garoua présente, d'une part, un tissu urbain structuré et, d'autre part, des quartiers spontanés et anarchiques (Foulbéré, Wouro Ourso, Souari, Koléré, Lidéré, Bibémiré, Demsaré, Kanadi...) très susceptibles aux inondations (source: Institut national de cartographie [Cameroun], image ASTER; réalisation: F. Saha).

de croissance démographique de l'ensemble de la population. En plus de l'accroissement naturel, la population urbaine est alimentée par les migrations, notamment l'exode rural. D'après Barbier et al. (1981), l'attrait du milieu urbain est lié à la possibilité de trouver les emplois rémunérés, le goût de l'aventure, la menace de certains maux sociaux tels que la sorcellerie dans les villages. En zone sahélienne confrontée à l'insécurité, les centres urbains (Mokolo, Kousséri, Maroua, etc.) sont des refuges pour les populations persécutées, notamment en zone frontalière. Grosso modo, les villes connaissent une densification démographique et s'étendent majoritairement par un processus de coalescence auréolaire (Mbaha et al., 2013). À Bamenda, les terres impropres à la construction (pentes abruptes et bas-fonds) accueillent un nombre croissant d'habitants (Nyambod, 2010). Autour des villes, on observe également le développement de fronts d'urbanisations privilégiant les terres les plus proches nonobstant les contraintes naturelles parfois très défavorables. Nassa Dabie (2010) remarque le besoin vital de chaque homme de justifier d'un toit privé, qui est un facteur d'élévation sociale. Dans cette quête de logements privés, plus de $75 \%$ de la population au Cameroun construit elle-même sa maison sans permis de bâtir, uniquement avec l'aide de la famille, des amis et des moyens très limités (Pettang, 1999).

\section{La pauvreté rampante}

Le Cameroun présente d'énormes limites dans la conduite de grands travaux de protection et même de suivi du processus d'urbanisation. Les inondations de 2012 dans les agglomérations le long du Logone (Yagoua, Pouss, Maga et Kousséri) étaient dues au manque d'entretien de la digue construite en 1982 sur cette rivière grâce à un partenariat avec l'Allemagne. La 


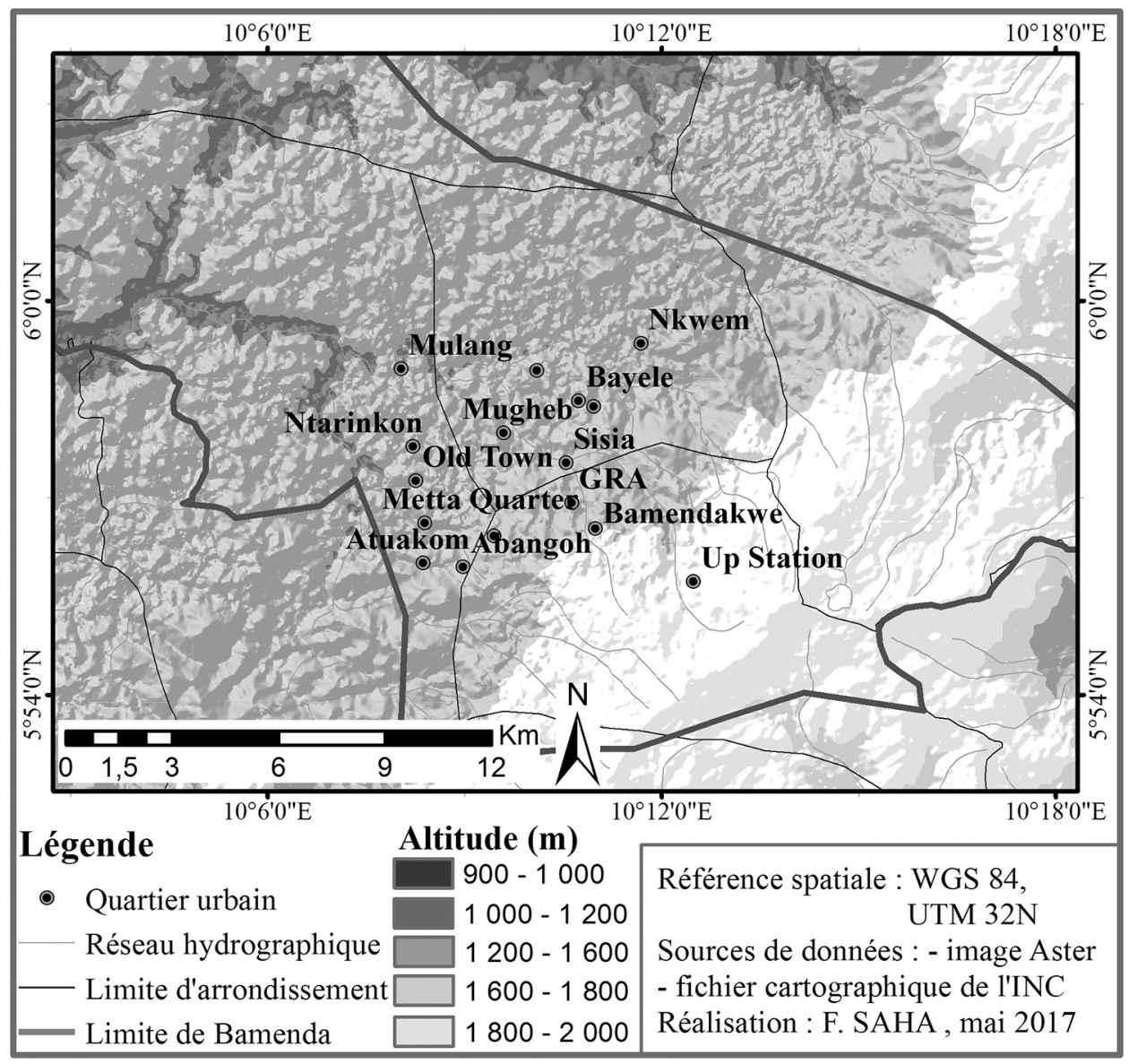

Fig. 3. Relief et contraintes du site de la ville de Bamenda.

La communauté urbaine de Bamenda présente une densité de $695 \mathrm{ha} / \mathrm{km}^{2}$ en moyenne (milieu rural et milieu urbain). Le centre de la ville est localisé dans un espace très accidenté et fortement escarpé. La rivière Mezam qui draine la ville au Nord se caractérise par des crues saisonnières qui inondent les quartiers en vallée (Mulang, Below Foncha, etc.). La récurrence des mouvements de masse est élevée en raison de l'occupation anarchique des pentes abruptes le long de l'escarpement, notamment dans les quartiers Sisia, Abangoh, New Layout...

(source: Institut national de cartographie [Cameroun], image ASTER; réalisation: F. Saha).

crise économique subite de la fin des années 1980 avait porté un sérieux coup aux capacités de l'État à mettre en œuvre sa politique sociale. La baisse du niveau de vie consécutive à la diminution des salaires avait entraîné une généralisation de la débrouillardise des citadins dans les différentes villes. En moyenne, l'incidence de la pauvreté au Cameroun était de $37,5 \%$ en 2014 . Cette situation a peu évolué; en 2001 c'est $40,2 \%$ de la population du Cameroun qui vivait en deçà du seuil de pauvreté, premier facteur de vulnérabilité face aux catastrophes naturelles (UNISDR, 2015).

La cohabitation entre différentes classes sociales (haute, moyenne et basse) dans les villes est observée dans la nomenclature urbaine et marquée par une forte discrimination entre les quartiers structurés et les quartiers de fait sans planification (Fig. 6). Les pauvres n'ayant pas de moyens de s'acheter des terrains sur des sites convenables se dirigent vers les zones à risque caractérisées par des parcelles moins coûteuses (Garry et Veyret, 1996). La cession du droit foncier dans ces milieux n'est pas encadrée, aucune norme de construction n'est respectée, la prise en compte des dessertes et la mise en place de réseaux d'assainissement sont défaillantes. Certains maires et délégués de gouvernement décrètent depuis quelques années la destruction de certains quartiers où les risques de catastrophe sont élevés. L'exemple du lieu-dit Fanta Citron au quartier Mvog-Ada est illustratif. En effet, en 2015 la communauté urbaine de Yaoundé a procédé à la destruction totale de ce quartier et les populations ont été orientées vers les périphéries de la ville.

La structuration des quartiers résidentiels est brouillée par la présence de ménages pauvres. Ils s'y installent pour profiter des opportunités d'emploi et parfois des largesses des riches comme à Lomé au Togo (Fiawumor, 2013). Il s'agit quelquefois de familles locales qui ont 


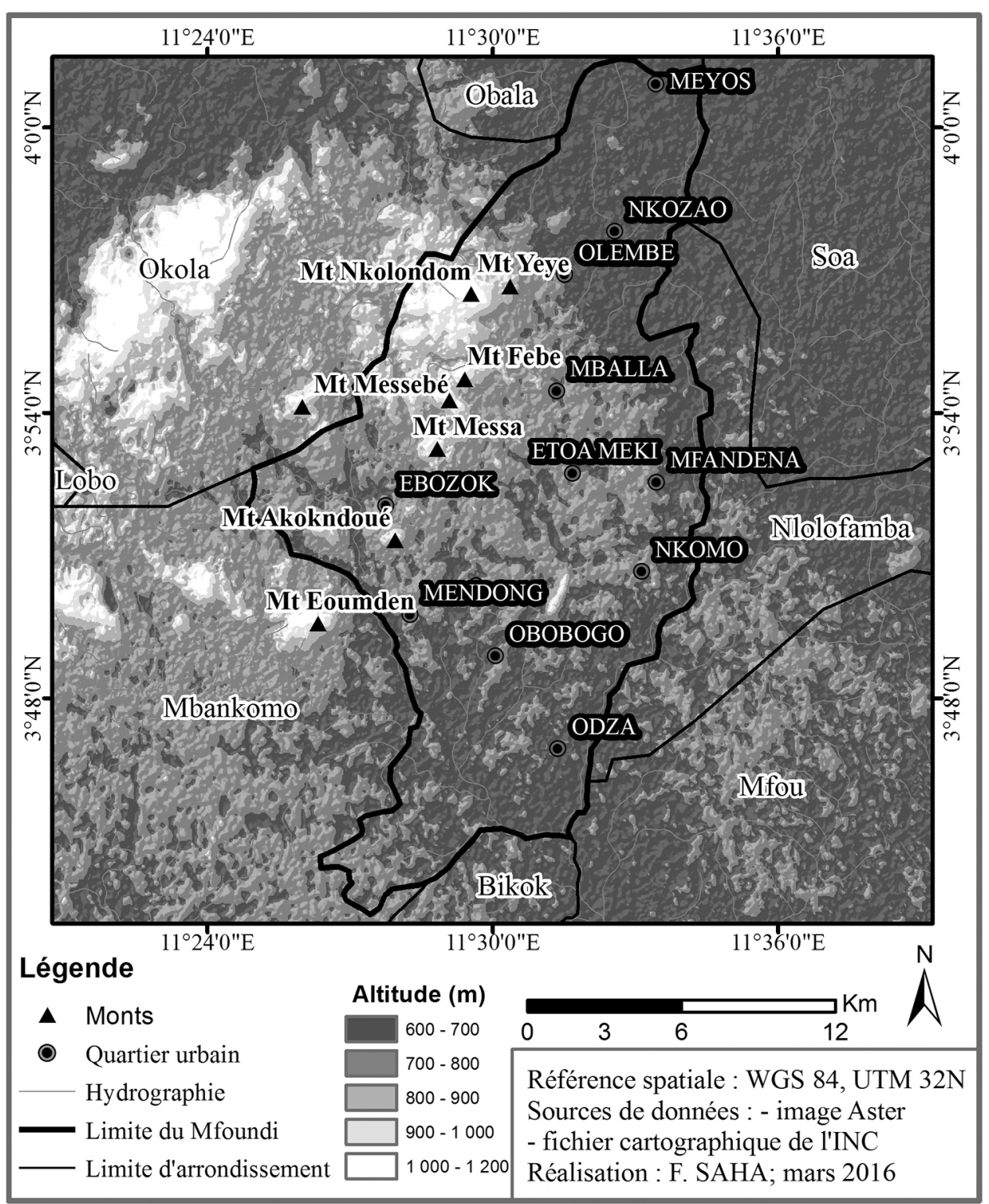

Fig. 4. Relief et contraintes du site de la ville de Yaoundé.

Yaoundé, capitale politique du Cameroun, compte environ 2,5 millions d'habitants selon une projection établie en 2005 à partir des chiffres du troisième recensement général de la population et de l'habitat (1 817524 hab). Baptisée ville aux sept collines en référence aux mornes en demi-orange qui ceinturent la ville, Yaoundé est un site d'aménagement difficile. L'alternance de pentes abruptes et de bas-fonds inondables explique la récurrence d'aléas hydroclimatiques et de mouvements de masse. Après le Schéma directeur d'aménagement et d'urbanisme de 1982, le développement de la ville est aujourd'hui encadré par un plan de déplacements urbains (PDU) horizon 2020. Les objectifs visés sont: réunifier et rééquilibrer la ville, la rendre plus accueillante, plus agréable à vivre et plus accessible pour favoriser son rayonnement (AUGEA et al., 2008). Dans cette mouvance, de nombreux quartiers (Vogt Ada, Mokolo, Oyom Abang, etc.) connaissent des travaux de restructuration ponctués par le déguerpissement des populations vivant dans les zones à risque (source: Institut national de cartographie [Cameroun], image ASTER; réalisation: F. Saha).

assisté à l'urbanisation de leur espace ancestral sans suivre les mutations socioéconomiques en cours. Ce constat de mixité sociospatiale est complété par la présence de familles aisées qui se font remarquer, dans les quartiers mal structurés, par la qualité de leurs constructions.

\section{La dégradation de l'environnement}

Les villes camerounaises présentent à ce jour les manifestations les plus patentes de la dégradation de l'environnement dans le triangle national. Les installations urbaines commandent parfois des aménagements 


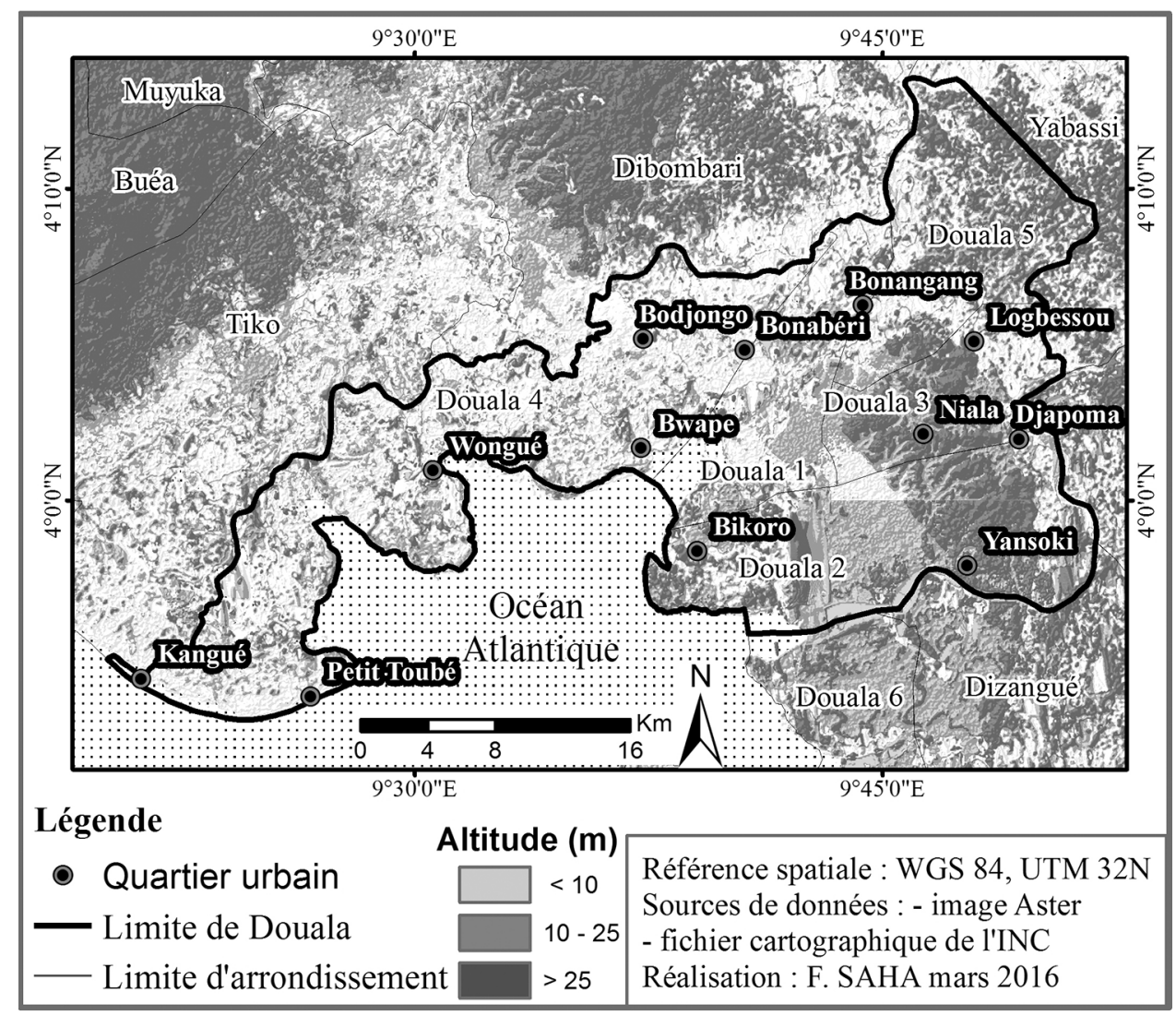

Fig. 5. Relief et contraintes du site de la ville de Douala.

Pôle industriel, la ville de Douala présente une densité moyenne de $2210 \mathrm{hab} / \mathrm{km}^{2}$. Il s'agit d'une ville côtière. Près de $40 \%$ de la superficie urbanisée est située à moins de $10 \mathrm{~m}$ d'altitude. Cette ville a toujours été confrontée au phénomène des inondations mais ce problème est aujourd'hui plus fréquent et plus violent. Les ravinements et les glissements de terrain sont enregistrés de plus en plus fréquemment. Les facteurs majeurs sont l'occupation anarchique de l'espace urbain, les dysfonctionnements et le manque de curage régulier du réseau de drainage. La réalité du changement climatique représente pour cette ville un facteur supplémentaire de risque comme pour l'ensemble des villes côtières du monde (GIEC, 2014)

[source: Institut national de cartographie (Cameroun), image ASTER; réalisation : F. Saha].

qui, mal maîtrisés, renforcent les menaces de catastrophes. Le cas d'aménagement des lits de cours d'eau peut être cité pour nombre de quartiers urbains au Cameroun. Les inondations répétitives du Mfoundi à Yaoundé sont l'œuvre du mauvais calibrage du lit de drainage souterrain traversant le centre de la ville. Il en est de même du Mayo-Kaliao à Maroua où les ponts sont souvent submergés en période de crue. Par ailleurs, l'élimination plus ou moins totale de la végétation en milieu urbain amplifie les risques morphodynamiques. À Douala, Olinga Olinga (2012) évoque les glissements de terrains comme une réponse de la nature aux pressions anthropiques. Le tableau 1 recense quelques cas d'événements impliquant les aléas naturels ayant affecté certaines grandes agglomérations du Cameroun pendant les deux dernières décennies.

On remarque une forte fréquence des inondations qui font des morts presque chaque année dans les villes du
Cameroun. L'événement de 2001 à Limbé fut causé par de très fortes pluies pendant plusieurs jours. Bamenda, Douala, Yaoundé sont les plus fréquemment touchées. Notons que de nombreuses catastrophes ayant eu lieu dans certaines villes du Cameroun ne sont pas enregistrées. En effet, il arrive que les populations victimes, conscientes de leur occupation illégale d'espaces à risques, acceptent les dégâts subis par crainte des représailles des autorités (Saha, 2013).

\section{Les changements climatiques}

Plusieurs manifestations pouvant être attribuées au réchauffement de l'atmosphère sont observables au Cameroun : augmentation des températures, élévation du niveau de la mer et perturbations pluviométriques. Ces changements sont à l'origine de nouveaux risques dans les villes camerounaises et les menaces existantes sont renforcées. 

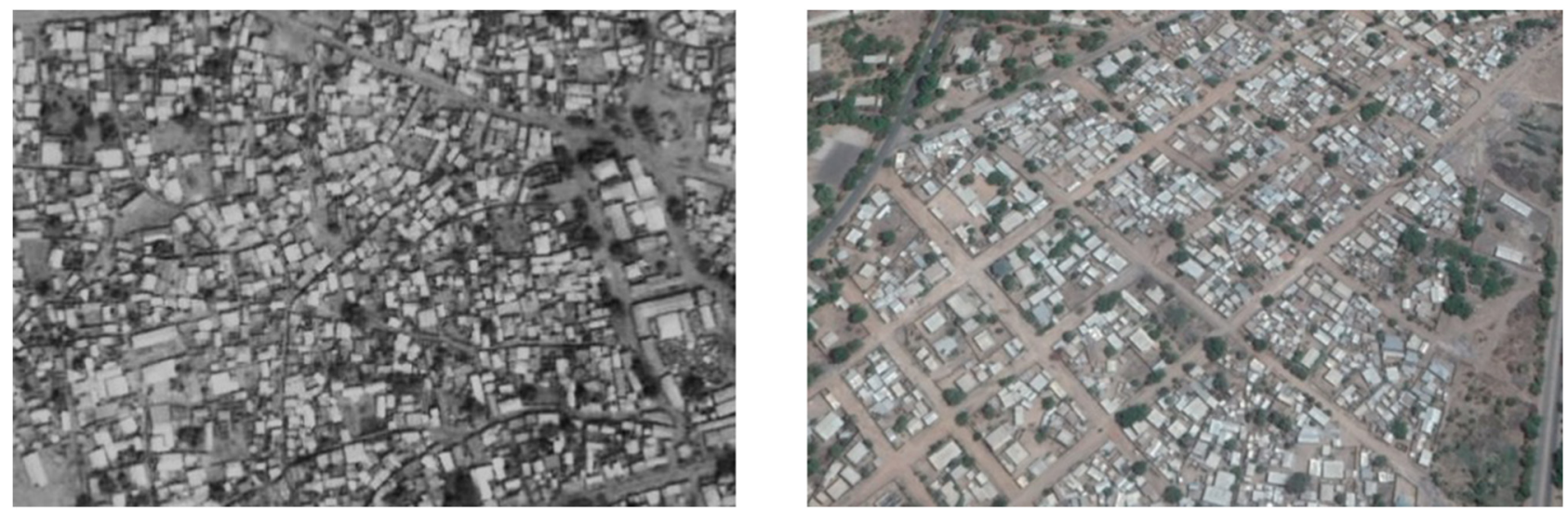

Fig. 6. Diversité du tissu urbain de Garoua.

À gauche le quartier Foulbéré : non structuré, entassement de l'habitat. À droite, le quartier Marouaré : tissu résidentiel et voirie assez bien organisée (@) Google Earth).

En Afrique, les observations du GIEC (2014) font état de l'augmentation de la sécheresse, d'une forte exposition à l'élévation du niveau de la mer et de la variabilité des précipitations sur tout le continent. Au Cameroun, une étude menée en 2008 par le Programme des Nations unies pour le développement (PNUD) relève l'augmentation des températures moyennes sur l'ensemble du territoire $\left(+0,7^{\circ} \mathrm{C}\right.$ de 1960 à 2007$)(\mathrm{McSweeney} \mathrm{et} \mathrm{al.,}$ 2008). Pour ce qui est des précipitations, la même étude note une baisse des totaux annuels, la zone sahélienne étant la plus touchée. Au niveau de la zone côtière, Douala, Kribi, Limbé... subissent l'élévation du niveau de la mer. Fonteh et al. (2009) font état d'une élévation de 1,8 à 2,2 $\mathrm{mm}$ par an entre 1948 et 2003. La recrudescence des inondations à Douala ces dernières années est une des conséquences de ce phénomène (Mbevo Fendoung, 2016). L'augmentation des températures affecte les zones de fortes concentrations humaines, en particulier les milieux urbains, l'ambiance thermique de référence des villes côtières et tropicales étant déjà caniculaire (MINEPDED, 2015).

La situation des précipitations résumée dans la moyenne des totaux annuels renferme plusieurs réalités sous-jacentes. Saha et Tchindjang (2017) relèvent une augmentation de $3,42 \%$ de la hauteur moyenne des précipitations entre la décennie 1961-1970 et la décennie 2001-2010 à Bamenda. Dans la même ville, les auteurs remarquent que le nombre annuel de jours pluvieux est en nette diminution et que certaines années la distribution saisonnière des pluies est perturbée. Ainsi les phénomènes de pluies extrêmes sont plus fréquents et se voient renforcés. Il en résulte des inondations et des coulées boueuses avec une perception de l'intensité et de la fréquence des risques fortement diminuée par la dynamique en cours. Un rapport de la GIZ (2013) ${ }^{6}$ relève une situation plus préoccupante pour les zones climatiques extrêmes du Cameroun, notamment la côte et la zone sahélienne où les variations interannuelles du climat renforcent les aléas et intensifient la vulnérabilité des populations.

\section{Les limites de la protection civile}

La protection civile est l'ensemble des moyens à mettre en œuvre pour assurer la protection de la population, des biens immobiliers et des moyens de production économique avant, pendant et après une situation de crise à grande échelle. Au Cameroun, il existe un Conseil national de la protection civile placé sous l'autorité du Secrétariat général de la présidence de la République. Il travaille en collaboration avec l'Observatoire national des risques (ONR) et la Direction de la protection civile (DPC) logée au ministère de l'Administration territoriale (MINAT). La DPC est chargée de l'organisation générale de la protection civile sur l'ensemble du territoire national. Elle gère les aides et les indemnisations en temps de crise et assure la coordination entre les administrations concernées.

Une fois de plus, un fossé existe entre ce qui est prévu et la gestion effective des catastrophes sur le terrain. Il est difficile d'apercevoir des initiatives préventives, notamment dans les zones exposées. Sur le plan opérationnel, seulement une quinzaine sur les cinquante-huit que compte le Cameroun disposent d'un plan opérationnel

\footnotetext{
${ }^{6} \mathrm{La}$ Gesellschaft für Internationale Zusammenarbeit (GIZ) est l'agence de coopération internationale allemande pour le développement.
} 
Tab. 1. Quelques cas de catastrophes naturelles dans les villes du Cameroun entre 1995 et 2010 (source: Buh Wung, 2009; Olinga Olinga, 2012; Saha, 2013; Tchio Nkemta, 2014).

\begin{tabular}{|c|c|c|c|c|}
\hline Année & Ville & Risque naturel & Dégâts corporels & $\begin{array}{l}\text { Dommages économiques } \\
\text { et structurels fréquents }\end{array}$ \\
\hline 1995 & Bamenda & Inondation & 2 morts & \multirow{19}{*}{$\begin{array}{l}\text { Aggravation ou déclenchement } \\
\text { de maladies hydriques } \\
\text { Destruction de bâtiments } \\
\text { Noyade d'animaux } \\
\text { Destruction d'équipements } \\
\text { industriels } \\
\text { Perte de stocks de ressources } \\
\text { alimentaires } \\
\text { Destruction de champs de culture } \\
\text { Pollution des sources d'eau } \\
\text { Destruction des routes et des ponts }\end{array}$} \\
\hline 1998 & Bamenda & Inondation & 3 morts & \\
\hline 1999 & Bamenda & Inondation & 1 mort & \\
\hline \multirow[t]{3}{*}{2000} & Bamenda & Inondation & 3 morts & \\
\hline & Maroua & Inondation & 4 morts & \\
\hline & Douala & Inondation & 2 morts & \\
\hline \multirow[t]{4}{*}{2001} & Bamenda & Inondation & 1 mort & \\
\hline & Douala & Inondation & 4 morts & \\
\hline & Maroua & Inondation & 2 morts & \\
\hline & Limbé & $\begin{array}{l}\text { Inondation } \\
\text { Glissement de terrain }\end{array}$ & $\begin{array}{l}30 \text { morts } \\
2000 \text { sans-abri }\end{array}$ & \\
\hline 2004 & Bamenda & Inondation & 1 mort & \\
\hline 2005 & Bamenda & Inondation & 1 mort & \\
\hline 2006 & Bamenda & Inondation & 2 morts & \\
\hline 2007 & Bamenda & $\begin{array}{l}\text { Inondation } \\
\text { Glissement de terrain }\end{array}$ & 3 morts & \\
\hline 2008 & Yaoundé & Inondation & 2 morts & \\
\hline \multirow[t]{3}{*}{2009} & Bamenda & $\begin{array}{l}\text { Inondation } \\
\text { Glissement de terrain }\end{array}$ & 5 morts & \\
\hline & Yaoundé & Inondation & $1 \mathrm{mort}$ & \\
\hline & Douala & $\begin{array}{l}\text { Inondation } \\
\text { Glissement de terrain }\end{array}$ & $\begin{array}{l}1 \text { mort } \\
3 \text { morts }\end{array}$ & \\
\hline 2010 & Bamenda & Inondation & 2 blessés & \\
\hline
\end{tabular}

d'organisation des secours (plan Orsec). Pourtant, la généralisation de cet instrument contribuerait, comme en France, à la territorialisation du risque pour une gestion de proximité (Reghezza-Zitt, 2015). Le manque de moyens financiers est aussi déploré. Le budget annuel d'intervention de la DPC s'élève à 500 millions de francs CFA, soit seulement $1,07 \%$ des besoins exprimés dans l'analyse des contours juridiques, institutionnels et financiers de l'Observatoire national des risques ainsi que du plan de contingence (DPC, 2003).

\section{Discussion}

Le constat de l'augmentation des risques naturels dans les grandes agglomérations du Cameroun met en relation plusieurs acteurs. Au niveau institutionnel, les communes et communautés urbaines sont interpellées dans la prévention par le suivi du processus d'urbanisation avec un respect strict des règles (acquisition des parcelles et normes de construction). L'État à travers les textes réglementaires et les institutions est aussi appelé à renforcer l'encadrement des communautés pour la sécurité des populations qui doivent être activement impliquées. Les déterminants qu'il faut prendre en compte pour atteindre cet objectif de prévention des catastrophes sont: la pauvreté, la dégradation de l'environnement, l'évolution démographique, le système foncier, la protection civile et la planification urbaine (Fig. 7).

D'un point de vue prévisionnel, il est difficile de maîtriser les perspectives d'extension spatiale à long terme des villes. Confrontées à des sites d'aménagement difficile, les villes camerounaises exigent une stratégie multisectorielle pour la prévention des catastrophes naturelles (Fogwe et Tchotsoua, 2007). La lutte contre la pauvreté est le principal défi sur le plan économique (UNISDR, 2015). Les institutions (communes, DPC et autres services sectoriels) ont besoin de plus de moyens pour contrôler le processus d'urbanisation (DPC, 2003). L'augmentation de l'offre en logements sociaux au profit des populations démunies est une solution contre l'extension en continu des bidonvilles. Il faut remarquer que cette perspective mérite d'être repensée pour les pays du Sud où les logements sociaux se sont révélés être de véritables gouffres économiques pour les États et les institutions financières (Biehler et al., 2015 ; Dao, 2016). 


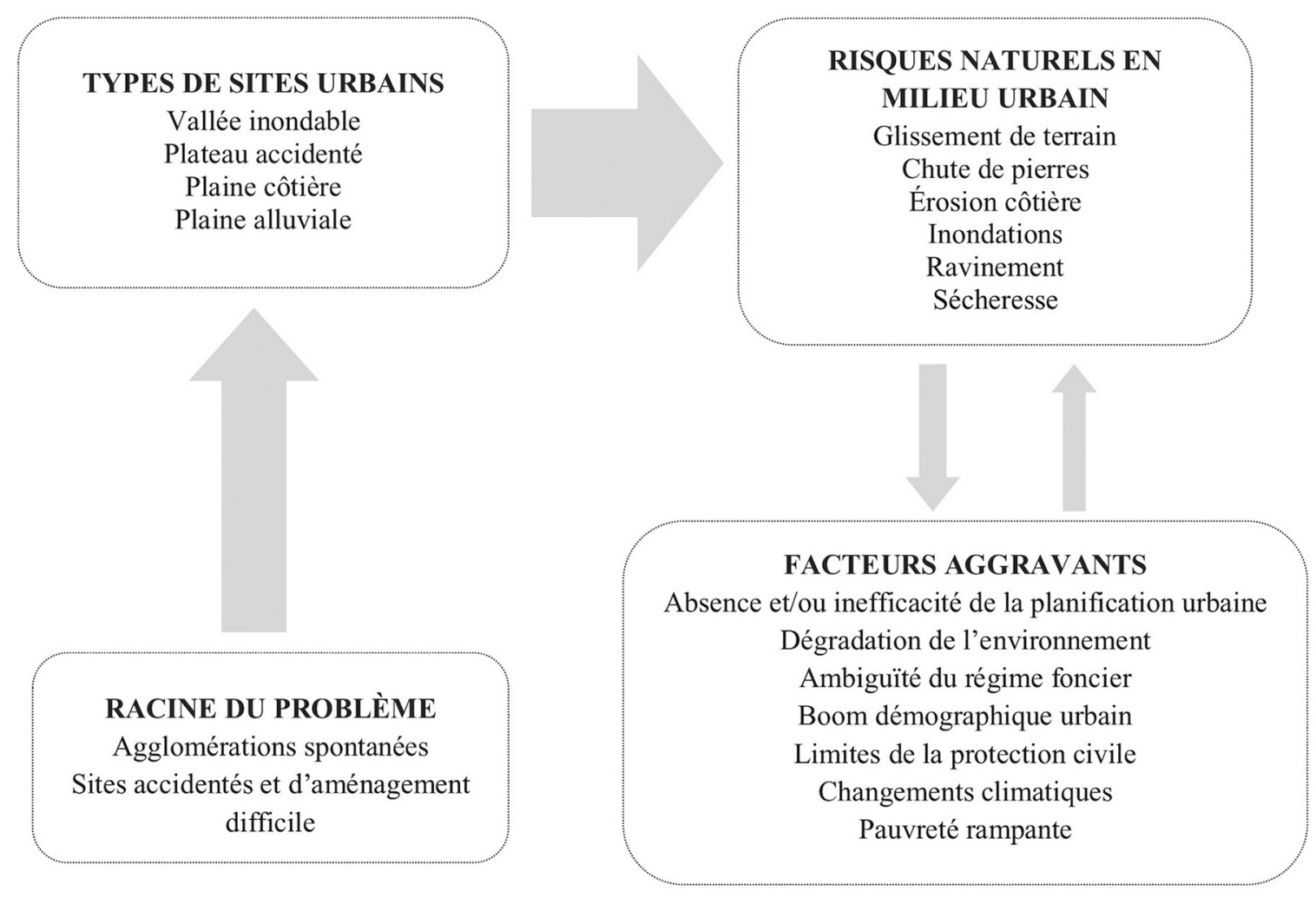

Fig. 7. Processus de production des risques naturels dans les villes camerounaises (réalisation: F. Saha).

L'encadrement de la production de lots serait un début de solution. L'état de l'ensemble des grandes agglomérations du Cameroun appelle aujourd'hui la poursuite d'actes courageux de rénovation et de restructuration. Les communautés urbaines de Douala, Yaoundé, Bafoussam se sont illustrées depuis quelques années par des démolitions, l'aménagement de sites et l'orientation de l'urbanisation vers les sites périphériques avec le développement du transport urbain. Le renforcement de la connaissance du risque par la sensibilisation des populations dans les quartiers à risques augmenterait la participation des ménages à la prévention des catastrophes. Il est vrai que le besoin d'un espace vital prime généralement sur les questions de sécurité. La mise en exergue de l'ampleur du danger appellerait à plus de méfiance. En outre, les populations pourraient être mises à contribution pour participer efficacement aux secours en cas de catastrophe. L'ambiguïté du système foncier donne aux acteurs coutumiers la possibilité de continuer d'attribuer des lots impropres à la construction où les populations s'installent anarchiquement (Njouonang Djomo, 2012). Cette réalité aggrave sans cesse la situation dans les villes et les mesures de remédiation se compliquent davantage.

Les populations devraient également être mises devant leurs responsabilités. Acteurs de leurs propres malheurs, elles sont responsables du renforcement des risques urbains. Une efficacité dans les actions de prévention ne sera obtenue que par une franche collaboration entre les autorités et les populations. Les associations de quartier pourraient jouer un rôle de police pour prévenir la dégradation de l'environnement et contribuer au contrôle de l'occupation du sol en collaboration avec les autorités (Castrillo Romón et Santos Y Ganges, 2008).

Loin d'être une situation de référence pour les villes prise en particulier, ce travail présente de manière générale les principaux facteurs de production des risques dits naturels dans les grandes agglomérations du Cameroun. Des exemples sont issus des recherches menées par le collectif des auteurs de cet article. Il est donc nécessaire pour chaque ville d'évaluer en fonction des réalités locales la place de chaque facteur dans la construction de sa vulnérabilité sociale et physique. Avec le quatrième recensement général de la population et de l'habitat déjà ordonné par les autorités compétentes, il sera nécessaire de mettre à jour les données démographiques avec des modifications sur l'armature urbaine du pays.

\section{Conclusion}

L'objectif de ce travail était d'évaluer les facteurs qui contribuent à la construction des risques dits naturels dans les grandes agglomérations du Cameroun. Il ressort des recherches menées sur quelques villes (Douala, Yaoundé, Bamenda, Garoua, Maroua) que l'augmentation des catastrophes est liée aussi bien aux facteurs 
naturels qu'aux facteurs anthropiques. Du point de vue naturel, le caractère fortement accidenté des sites urbains, la localisation sur la côte maritime, la localisation de certaines villes en milieu tropical chaud et la forte densité du réseau hydrographique sont les principaux facteurs qui expliquent l'exposition des centres urbains camerounais aux risques.

Exposées aux risques naturels de par leur situation géographique, les populations des villes le sont d'abord principalement du fait de la pauvreté économique. Du côté des autorités, l'incapacité financière, humaine et technique à planifier à long terme le processus d'urbanisation favorise l'extension spatiale des villes de fait où le spontané l'emporte sur le normatif. De plus, le système de protection civile présente de nombreuses failles liées à l'absence d'initiatives de prévention et au manque d'équipements. Au niveau des individus, l'incapacité à acquérir des parcelles aedificandi et l'impéritie à construire des logements décents dans le respect des normes de constructions et d'urbanisation sont à l'origine des installations dans les bas-fonds et les pentes escarpées. La dégradation de l'environnement (déforestation, modification du système hydrographique, pollutions, dégradation des terres...) liée à la forte pression démographique exacerbe les risques d'inondation, de glissements de terrains, de chutes de pierres et de sécheresse dans les villes. La menace des changements climatiques, hautement imprévisible, complique les plans d'aménagement et porte un sérieux coup aux moyens de subsistance des populations. Il est vrai qu'il serait naïf de prétendre au risque zéro (Pigeon, 2005), mais il reste encore beaucoup à faire de la part de l'ensemble des acteurs (populations, autorités et partenaires au développement) pour prévenir l'augmentation des risques de catastrophe dans les centres urbains du Cameroun et y apporter des solutions.

\section{Remerciements}

Nous exprimons notre profonde gratitude au comité de rédaction de Natures Sciences Sociétés et surtout aux relecteurs anonymes. Les avis sur les versions successives de notre article nous ont permis d'éviter des confusions conceptuelles et d'améliorer la contextualisation du sujet.

\section{Références}

Anougue Tonfack B.F., Betguiro Ngwaibo F., Haiwe B.R., Nwowe W., Tchokolva P., Wadai D., 2013. Rapport de l'étude sur la vulnérabilité des communes de la région de l'Extrême-Nord aux effets du changement climatique, Maroua, Gesellschaft für Internationale Zusammenarbeit (GIZ)-ProPSFE Extrême-Nord.
Antoine P., 1990. Croissance urbaine et insertion des migrants dans les villes africaines, in Actes du colloque international Des langues et des villes, 15-17 décembre, Dakar, Niamey, Agence de coopération culturelle et technique.

Assako Assako R.J., 1995. Amélioration de l'habitat à Yaoundé : succès tardif ou fiasco consommé?, Histoire et Anthropologie, 11, 44-50.

Assako Assako R.J., 1997. Apport des systèmes d'information géographique dans l'analyse des risques d'inondation et de glissement de terrain à Yaoundé, in Bley D., Champaud J., Baudot P., Brun B., Pagezy H., Vernazza-Licht N. (Eds), Villes $d u$ Sud et environnement, Châteauneuf-Grasse, Éditions de Bergier, 110-124.

AUGEA International, IRIS Conseil, ARCAUPLAN, 2008. Yaoundé 2020. Plan directeur d'urbanisme. Rapport de présentation, Yaoundé, ministère du Développement urbain et de l'Habitat, Cameroun, communauté urbaine de Yaoundé.

Baird A., O’Keefe P., Westgate K., Wisner B., 1975. Towards an explanation and reduction of disaster proneness, Disaster Research Unit, University of Bradford Occasional Papers, 11.

Barbier J.-C., Courade G., Gubry P., 1981. L'exode rural au Cameroun, Cahiers Orstom. Série Sciences Humaines, 18, 1, 107-147.

Biehler A., Choplin A., Morelle M., 2015. Le logement social en Afrique: un modèle à (ré)inventer?, Métropolitiques, www.metropolitiques.eu/Le-logement-social-en-Afriqueun.html.

Bouabre Gnoka M., 2013. Développement urbain et menace d'érosion côtière à Abidjan, European Scientific Journal, 9, 29, 55-71.

BUCREP (Bureau central des recensements et des études de population, Cameroun), 2010. Troisième recensement général de la population et de l'habitat. Rapport de présentation des résultats définitifs, Yaoundé, Cameroun.

Buh Wung G., 2009. Geographic information systems based demarcation of risk zones: the case of the Limbe SubDivision, Journal of Disaster Risk Studies, 2, 1.

Burton I., Kates R.W., White G.F., 1978. The environment as hazard, New York, Oxford University Press.

Cameroon Engineering, 2008. Plan directeur d'urbanisme de la ville de garoua. Rapport diagnostic et rapport justificatif et règlement d'urbanisme, Garoua, communauté urbaine de Garoua.

Castrillo Romón M.A., Santos Y Ganges L., 2008. Urbanisme et militantisme de quartier dans les quartiers populaires de Valladolid, Espaces et sociétés, 134, 3, 53-66.

Dao B., 2016. Les difficultés liées au financement du logement social en Afrique. Communication à la $49 \mathrm{e}$ conférence du réseau Habitat et francophonie (RHF), Le logement social à l'aune de la conférence ONU-Habitat III. Quelles perspectives pour les pays francophones?, 30 mai1erjuin, La Réunion, http://habitatfrancophonie.org/wpcontent/uploads/2016/04/Difficult\%C3\%A9s-li\%C3\% A9es-au-financement-du-Logement-social-en-AfriqueBaba-Dao.pdf.

DPC (Direction de la protection civile, Cameroun), 2003. Prévention des risques et gestion des catastrophes au 
Cameroun: contribution de la protection civile $d u$ Cameroun à la conférence des catastrophes de Kobe (Japon), World Disaster Reduction Campaign, ISDR.

Fiawumor S., 2013. Togo : Lomé, un exemple de mixité sociale et résidentielle à l'africaine. Note de terrain.

Fogwe Z.N., Tchotsoua M., 2007. Évaluation géographique de deux décennies de lutte contre les inondations dans la ville de Douala (Cameroun), in Actes des Journées scientifiques inter-réseaux de l'Agence universitaire de la francophonie (JSIRAUF), 6-9 novembre, Hanoi.

Fombe L.F., Balgah S.N., 2010. The urbanisation process in Cameroon. Patterns, implications and prospects, New York, Nova Science Publishers.

Fonteh M., Esteves L.S., Gehrels W.R., 2009. Mapping and valuation of ecosystems and economic activities along the coast of Cameroon: implications of future sea level rise, Coastline Reports, 13, 47-63.

Franqueville A., 1984. Yaoundé. Construire une capitale, Bondy, Orstom.

Garry G., Veyret Y., 1996. La prévention du risque d'inondation: l'exemple français est-il transposable aux pays en développement?, Cahiers des sciences humaines, 32, 2, 423-443.

GIEC, 2014. Changements climatiques 2014. Rapport de synthèse, contribution des groupes de travail I, II et III au cinquième rapport d'évaluation $d u$ Groupe d'experts intergouvernemental sur l'évolution du climat [Pachauri R.K., Meyer L. (Eds)], Genève, GIEC.

GIZ (Gesellschaft für Internationale Zusammenarbeit), 2013. Rapport d'étude sur le changement climatique, la dynamique démographique et la santé de reproduction dans les régions du Sud-Ouest et de l'Extrême Nord du Cameroun, Yaoundé, Cameroun.

Guha-Sapir D., Hoyois P., Below R., 2014. Annual disaster statistical review 2013. The numbers and trends, Brussels, Centre for Research on the Epidemiology of Disasters (CRED).

Hossenlopp J., 1971. Évolution de l'urbanisation dans 14 États d'Afrique noire et Madagascar avec une esquisse prospective à l'horizon 1985, Cahiers Orstom. Série Sciences Humaines, 8, 1, 25-36.

Ledoux B., 1995. Les catastrophes naturelles en France, Paris, Payot.

Martin P., 2006. Ces risques que l'on dit naturels, Paris, Eyrolles.

Mbaha J.-P., Olinga J.M., Tchiadeu G. 2013. Cinquante ans de conquête spatiale à Douala: d'héritage colonial en construction à patrimoine socio-spatial vulnérable aux risques naturels, in Oyono D. (Ed.), Actes du colloque du cinquantenaire de la réunification du Cameroun, 13-14 février, Douala, Douala, Université de Douala, 415-435.

Mbevo Fendoung P., 2016. Analyse de la vulnérabilité et des stratégies locales d'adaptation aux changements climatiques en zone côtière camerounaise: cas de Cap Cameroun dans l'arrondissement de Douala 6e. Mémoire de master en géographie, Yaoundé, Université de Yaoundé 1.
McSweeney C., New M., Lizcano G., 2008. UNDP climate change country profiles. Cameroon, www.geog.ox.ac.uk/ research/climate/projects/undp-cp/.

Metzger P., D'Ercole R., 2011. Les risques en milieu urbain : éléments de réflexion, EchoGéo, 18, doi: 10.4000/echo geo. 12640 .

MINEPDED (Ministère de l'Environnement, de la Protection de la nature et du Développement durable, Cameroun), 2015. Plan national d'adaptation aux changements climatiques $d u$ Cameroun. Document préparé avec le soutien du Japon, la GIZ, le GWP et le PNUD, Yaoundé, MINEPDED.

Mounganga M.-D., 2009. Érosion côtière et risques littoraux face aux changements climatiques: essai d'analyses comparatives des indicateurs de vulnérabilité à Libreville (Gabon) et Pointe-Noire (Congo). Communication au colloque international du Secrétariat international francophone pour l'évaluation environnementale (SIFEE) Changements climatiques et évaluation environnementale, 26-29 mai, Niamey.

Mveng E., 1984. Histoire du Cameroun. Tome II, Yaoundé, CEPER.

Nassa Dabie D.A., 2010. Contraintes spatiales dans les mégalopoles africaines et risques naturels, in AlokoN'Guessan J., Diallo A., Motcho H.K. (Eds), Villes et organisation de l'espace en Afrique, Paris, Kartala.

Njouonang Djomo H., 2012. Stratégies d'accès au foncier face aux compétitions et mutations foncières dans la ville de Yaoundé : cas de l'arrondissement de Yaoundé VI. Mémoire de master, Yaoundé, Université de Yaoundé 1.

November V., 1994. Risques naturels et croissance urbaine: réflexion théorique sur la nature et le rôle du risque dans l'espace urbain, Revue de géographie alpine, 82, 4, 113123.

Nyambod E., 2010. Environmental consequences of rapid urbanisation: Bamenda City, Cameroon, Journal of Environmental Protection, 1, 1, 15-23.

Olinga Olinga J.M., 2012. Vulnérabilité des espaces urbains et stratégies locales de développement durable: étude du cas de la ville de Douala (Cameroun). Mémoire de master, Douala, Université de Douala.

Olvera L.D., Plat D., Pochet P., 2002. Étalement urbain, situations de pauvreté et accès à la ville en Afrique subsaharienne: l'exemple de Niamey, in Bussière Y., Madre J.-L. (Eds), Démographie et transport: villes du Nord et villes $d u$ Sud, Paris, L'Harmattan, 147-175.

Ould Sidi Cheikh M.A., Ozer P., Ozer A., 2007. Risques d'inondations dans la ville de Nouakchott (Mauritanie), Geo-Eco-Trop, 31, 19-42.

Pettang C., 1999. Diagnostic de l'habitat urbain au Cameroun. Aménagement urbain. Volume II. Éléments d'optimisation de la production d'un habitat urbain décent au Cameroun, Yaoundé, Presses universitaires de Yaoundé.

Pigeon P., 2005. L'efficacité des politiques gérant les risques dit naturels en France : lecture géographique, in Leone F., Vinet F. (Eds), La vulnérabilité des sociétés et des territoires face aux menaces naturelles: analyses géogra- 
phiques, Montpellier, Publications de l'Université PaulValéry-Montpellier 3, 35-27.

PNUD (Programme des Nations unies pour le développement), 2016. Rapport sur le développement humain 2016. Le développement humain pour tous, New York, UNDP.

Reghezza-Zitt M., 2015. Territorialiser ou ne pas territorialiser le risque et l'incertitude. La gestion territorialisée à l'épreuve du risque d'inondation en Île-de-France, $L$ 'Espace Politique, 26, 2.

Saha F., 2013. La vulnérabilité aux risques naturels en milieu urbain. Cas de la ville de Bamenda. Mémoire de master en dynamique de l'environnement et des risques, Yaoundé, Université de Yaoundé 1.

Saha F., Tchindjang M., 2017. Rainfall variability and floods occurrence in the city of Bamenda (northwest of Cameroon), Present Environment and Sustainable Development, 11, 1, 65-82.

Salem G., 1995. Urbanisation et santé en Afrique de l'Ouest. Géographie d'un petit espace dense: Pikine (Sénégal). Mémoire d'habilitation à diriger des recherches, Montpellier, Université Paul-Valéry-Montpellier 3.

Santos M., 1971. Les villes du Tiers monde, Paris, M.-T. Génin.

Savane L., Antoine P., 1989. La croissance des grandes villes en Afrique de l'Ouest. Exemples de Dakar, Abidjan et Nouakchott, in Actes de la 47 e session de l'Institut international de statistique, 29 août-6 septembre, Paris.

Suchel J.-B., 1988. Les climats du Cameroun. Thèse d'État, Saint-Étienne, Université de Saint-Étienne.

Tchekote H., Kaffo C., 2012. Déguerpissements et gouvernance urbaine: Yaoundé entre échec de planification et tentatives de régulation territoriale, Revue des Hautes Terres, 2, 1, 93-104.

Tchindjang M., 2012. Paradoxes et risques dans les hautes terres camerounaises: multifonctionnalité naturelle et sous valorisation humaine. Mémoire d'habilitation à diriger des recherches, vol. 3, Paris, Université ParisDiderot.

Tchio Nkemta D., 2014. Aménagement des quartiers à habitat précaire de Yaoundé Ve : cas de Mvog-Ada au lieu-dit Fanta Citron. Mémoire de master en urbanisme, Yaoundé, Université de Yaoundé 1.

Tchotsoua M., 2007. Les risques morpho-hydrologiques en milieu urbain tropical: cas de Yaoundé au Cameroun, in Actes des Journées scientifiques inter-réseaux de l'Agence universitaire de la francophonie (JSIRAUF), 6-9 novembre, Hanoi.

Thouret J.-C., D’Ercole R., 2009. Vulnérabilité aux risques naturels en milieu urbain: effets, facteurs et réponses sociales, Cahiers des sciences humaines, 32, 2, 407-422.

UNISDR (United Nations International Strategy for Disaster Reduction), 2015. Réduction du risque de catastrophe: bilan mondial, Genève, Nations unies.

Veyret Y., Meschinet de Richemond N., 2003. Les risques naturels dans les pays tropicaux : réflexions issues des travaux récents, in David G. (Ed.), Espaces tropicaux et risques. Du local au global, Orléans, Presses universitaires d'Orléans, 25-36.

Voundi E., 2016. Analyse et caractérisation des risques naturels en milieu périurbain: le cas du Mont Fébé. Mémoire de master en dynamique de l'environnement et des risques, Yaoundé, Université de Yaoundé 1.

Citation de l'article : Saha F., Tchio Nkemta D., Tchindjang M., Voundi É., Mbevo Fendoung P., 2018. Production des risques dits «naturels» dans les grands centres urbains du Cameroun. Nat. Sci. Soc. 26, 4, 418-433. 